\title{
Sarcomas de partes blandas en pacientes pediátricos. Análisis de una serie de casos del subtipo no rabdomiosarcoma
}

\author{
Pediatric soft tissue sarcomas. Analysis of non rhabdomyosarcoma group
}

\author{
Dr. Germán Luis Farfalli ${ }^{a}$ Dra. Ángeles Iriberrya ${ }^{a}$ Dr. José Ignacio Albergo ${ }^{a}$, Dr. Miguel Ángel Ayerza ${ }^{a}$, \\ Dr. Domingo Luis Muscolo y Dr. Luis Alberto Aponte-Tinao ${ }^{a}$
}

\section{RESUMEN}

Seevaluó una serie de 29 pacientes con diagnóstico de sarcomas de partes blandas variante no rabdomiosarcomas tratados quirúrgicamente entre 2000 y 2010, y se analizóla supervivencia global y los factores que influyen en el pronóstico. La edad media fue de 11,6 años (rango de 3 meses-17 años); 16 pacientes eran de sexo masculino y el tiempo promedio de seguimiento fue de 56 meses (de 8 a 132 meses). Se documentaron 8 variedades histológicas diferentes de tumores malignos, y el sarcoma sinovial resultó ser el más frecuente (14 pacientes). La cirugía de conservación de miembro fue posible en 28 pacientes y se asoció tratamiento adyuvante en 26 . La supervivencia global de la serie estudiada fue de $72 \%$ a los 5 años y, en 9 pacientes, se diagnosticó una recurrencia local. La presencia de metástasis $(p<0,0001)$ y la recurrencia local $(p<0,007)$ resultaron ser factores de pronóstico negativo para la supervivencia global. Palabras clave: sarcomas de partes blandas no rabdomiosarcomas, supervivencia, recurrencia local.

\section{ABSTRACT}

We evaluated 29 patients with non rhabdomyosarcoma soft tissue sarcomas treated with surgery between 2000 and 2010; we analyzed overall survival and which factors affect the prognosis. The mean age was 11.6 years (range 3 months- 17 years); 16 patients were males and the median follow-up was 56 months (8 to 132 months). Eight different histological malignant tumors were identified, being synovial sarcoma the most prevalent one (14 patients). Twenty-eight patients were treated with limb salvage surgery and in 26 cases, adjuvant therapy was used. Five years overall survival was $72 \%$. Nine of the 29 patients presented a local recurrence. The presence of metastases $(p<0.0001)$ and local recurrence $(p<0.007)$ were negative prognostic factors for overall survival.

Key words: non rhabdomyosarcoma soft tissue sarcomas, overall survival, local recurrence.

http:/ /dx.doi.org/10.5546/aap.2014.e257

a. Ortopedia y Traumatología - Sector de Ortopedia Oncológica y Trasplantes Óseos.

Hospital Italiano de Buenos Aires.

Correspondencia:

Dr. José Ignacio Albergo: nachoalbergo@hotmail.com.

Conflicto de intereses: ninguno que declarar.

Recibido: 26-12-2013

Aceptado: 31-7-2014

\section{INTRODUCCIÓN}

Los sarcomas pediátricos de partes blandas representan el $7 \%$ de todos los tumores malignos en pacientes menores de 18 años. ${ }^{1}$ Se dividen en dos grandes grupos: rabdomiosarcomas (RMS) y no rabdomiosarcomas (NRMS), y se caracterizan por tener un mejor pronóstico que los tumores de partes blandas del adulto. ${ }^{2-4}$ En particular, los tumores NRMS representan un grupo heterogéneo de tumores malignos derivados de células mesenquimales y han sido escasamente reportados en la literatura como entidad única, debido a su baja frecuencia y heterogeneidad. 5

La localización central es un factor pronóstico negativo, que presenta un peor índice de supervivencia y una mayor tasa de recurrencia local en comparación con los tumores de las extremidades. $^{7}$

El pilar fundamental del tratamiento para los sarcomas de partes blandas pediátricos es la resección quirúrgica en bloque, con márgenes libres de tumor. ${ }^{8}$ La terapia adyuvante con radioterapia, generalmente indicada en la población adulta para el control local de la enfermedad, suele no ser utilizada como rutina debido a las potenciales complicaciones a largo plazo que puede inducir, tales como alteraciones del crecimiento o desarrollo de tumores secundarios. ${ }^{5}$ El objetivo de este trabajo es evaluar la supervivencia global y determinar los factores que influyen en el pronóstico de un grupo de pacientes pediátricos, tratados quirúrgicamente en una misma institución, con diagnóstico de sarcomas de partes blandas tipo NRMS.

\section{CASOS CLÍNICOS}

Se realizó una búsqueda retrospectiva en nuestra base de datos oncológica entre enero de 2000 y enero de 2010, y se identificaron 29 pacientes menores de 18 años con diagnóstico de sarcomas de partes blandas del subtipo NRMS. Dieciséis eran de sexo masculino. La edad media fue de 11,6 años (rango de 3 meses-17 años) y la 
mediana de seguimiento, de 56 meses (de 8 a 132 meses). Todos los pacientes fueron estadificados inicialmente con radiografías, tomografías de tórax, abdomen y pelvis, y resonancia magnética nuclear del sitio afectado. En los 29 casos, se realizó punción biopsia bajo tomografía y con anestesia general en los pacientes menores de 16 años. La recomendación es biopsia incisional, pero puede ser apropiada la biopsia guiada por tomografía axial computada (TAC) o ecografía, con tru-cut. No es apropiado realizar el diagnóstico con aguja fina, ya que es necesario tener material suficiente para el diagnóstico histológico y molecular porque, muchos de estos subtipos histológicos, tienen alteraciones citogenéticas y moleculares características, determinadas por FISH y PCR. La biopsia debe ser siempre realizada por el equipo médico que llevará a cabo el tratamiento quirúrgico debido a que el lugar anatómico donde se realiza es muy relevante porque el tracto de esta debe ser siempre resecado en el acto quirúrgico. Es de suma importancia realizar buena hemostasia y evitar los hematomas posoperatorios para evitar la diseminación del tumor. El diagnóstico fue confirmado por histología. Se documentaron 8 variedades histológicas diferentes de tumores malignos, y el sarcoma sinovial resultó ser el más frecuente (14 casos) y, en segundo lugar, el fibrosarcoma (2 congénitos y 5 del adulto) (7 casos). Todos los pacientes recibieron tratamiento quirúrgico. En 28 casos, se realizó una resección en bloque asociada a una cirugía de conservación de miembro y, en 1 paciente, una amputación infrapatelar. Veintiséis pacientes recibieron, a su vez, algún tipo de adyuvancia (Tabla 1).

TABLA 1. Características demográficas y oncológicas de los pacientes

\begin{tabular}{|c|c|c|c|c|c|c|c|}
\hline Pacientes & Edad & Diagnóstico & Localización & Cirugía & T. adyuvante & Seguimiento & Metástasis \\
\hline 1 & 15 años & Fibrosarcoma & Pierna & CCM & RDT & 89 meses & $\mathrm{NO}$ \\
\hline 2 & 8 años & Fibrosarcoma & Pelvis & CCM & Quimio + RDT & 28 meses & NO \\
\hline 3 & 3 meses & Fibrosarcoma & Pie & Amp & Quimio & 54 meses & $\mathrm{NO}$ \\
\hline 4 & 8 meses & Fibrosarcoma & Muslo & CCM & Quimio & 31 meses & $\mathrm{NO}$ \\
\hline 5 & 12 años & Fibrosarcoma & Pierna & $\mathrm{CCM}$ & Quimio & 101 meses & $\mathrm{NO}$ \\
\hline 6 & 13 años & Fibrosarcoma & Brazo & CCM & Quimio & 119 meses & $\mathrm{NO}$ \\
\hline 7 & 2 años & Fibrosarcoma & Muslo & CCM & Quimio & 43 meses & SI \\
\hline 8 & 17 años & Leiomiosarcoma & Pierna & CCM & $\mathrm{NO}$ & 43 meses & $\mathrm{NO}$ \\
\hline 9 & 16 años & Leiomiosarcoma & Muslo & CCM & Quimo + RDT & 34 meses & SI (i) \\
\hline 10 & 17 años & Liposarcoma & Hombro & CCM & $\mathrm{NO}$ & 111 meses & $\mathrm{NO}$ \\
\hline 11 & 12 años & Sarcoma alveolar & Muslo & CCM & Quimio & 11 meses & $\mathrm{NO}$ \\
\hline 12 & 17 años & Sarcoma celulas claras & Pie & CCM & $\mathrm{NO}$ & 74 meses & $\mathrm{NO}$ \\
\hline 13 & 16 años & Sarcoma sinovial & Pierna & CCM & RDT & 96 meses & $\mathrm{NO}$ \\
\hline 14 & 17 años & Sarcoma sinovial & Glúteo & CCM & Quimio + RDT & 44 meses & $\mathrm{NO}$ \\
\hline 15 & 15 años & Sarcoma sinovial & Muñeca & CCM & Quimio & 24 meses & $\mathrm{NO}$ \\
\hline 16 & 13 años & Sarcoma sinovial & Muslo & CCM & Quimio & 48 meses & $\mathrm{NO}$ \\
\hline 17 & 11 años & Sarcoma sinovial & Pierna & CCM & Quimio + RDT & 132 meses & $\mathrm{NO}$ \\
\hline 18 & 14 años & Sarcoma sinovial & Región inguinal & CCM & Quimio + RDT & 130 meses & $\mathrm{NO}$ \\
\hline 19 & 15 años & Sarcoma sinovial & Glúteo & CCM & Quimio + RDT & 24 meses & $\mathrm{NO}$ \\
\hline 20 & 13 años & Sarcoma sinovial & Hueco poplíteo & CCM & Quimio & 26 meses & $\mathrm{NO}$ \\
\hline 21 & 12 años & Sarcoma sinovial & Muslo & CCM & Quimio & 8 meses & $\mathrm{NO}$ \\
\hline 22 & 3 meses & Sarcoma sinovial & Muslo & $\mathrm{CCM}$ & Quimio & 57 meses & $\mathrm{NO}$ \\
\hline 23 & 7 años & Sarcoma sinovial & Antebrazo & CCM & Quimio & 41 meses & $\mathrm{NO}$ \\
\hline 24 & 14 años & Sarcoma sinovial & Pierna & CCM & Quimio & 124 meses & $\mathrm{NO}$ \\
\hline 25 & 14 años & Sarcoma sinovial & Antebrazo & CCM & Quimio + RDT & 24 meses & SI (i) \\
\hline 26 & 11 año & Sarcoma sinovial & Muslo & $\mathrm{CCM}$ & Quimio & 65 meses & $\mathrm{NO}$ \\
\hline 27 & 15 años & Tumor desmoplásico & Lumbar & CCM & Quimio + RDT & 13 meses & SI \\
\hline 28 & 17 años & TMVNP & Pelvis & CCM & Quimio + RDT & 21 meses & SI \\
\hline 29 & 12 años & TMVNP & Pelvis & CCM & Quimio & 12 meses & SI \\
\hline
\end{tabular}

Amp: amputación; CCM: cirugía para conservación de miembro; Quimio: quimioterapia; RDT: radioterapia;

TMVNP: tumor maligno de la vaina del nervio periférico; i: metástasis al momento del diagnóstico. 
Se analizó la supervivencia con el método de Kaplan-Meier. Los factores pronósticos fueron evaluados mediante regresión LOG Rank. Valores de la $\mathrm{p}$ menores de 0,05 fueron interpretados como estadísticamente significativos.

La supervivencia global de la serie estudiada fue de $72 \%$ a los 5 años (CI 95\%: 56\%-90\%) (Figura 1). Dos pacientes presentaban metástasis al momento del diagnóstico y 4 las desarrollaron luego del tratamiento quirúrgico. De esos 6 pacientes, dos con metástasis pulmonares fueron tratados con resecciones quirúrgicas y tratamiento adyuvante. Al momento del último control, 5 de los 6 pacientes habían fallecido y solo uno sobrevive sin evidencia de enfermedad.

Con respecto a los márgenes posoperatorios, en 7 casos, se han obtenido márgenes amplios; en 19 pacientes, resultaron marginales; y en 3 , intralesionales. Nueve de los 29 pacientes presentaron una recurrencia local (1 paciente con márgenes amplios, 5 con márgenes marginales y los 3 pacientes con resecciones intralesionales). Todos fueron tratados con una nueva resección oncológica, y se asoció, en 5 casos, radioterapia. No hemos podido encontrar asociación estadísticamente significativa entre márgenes posoperatorios y recidiva local $(\mathrm{p}>0,05)$ (Figura 2).

La asociación entre recidiva local y el desarrollo de metástasis resultó estadísticamente significativa $(\mathrm{p}<0,001)$. A su vez, la presencia de metástasis $(p<0,0001)$, así como la recurrencia local ( $p<0,007)$, fueron factores pronósticos negativos para la supervivencia global.

FIGURA 1. Supervivencia global

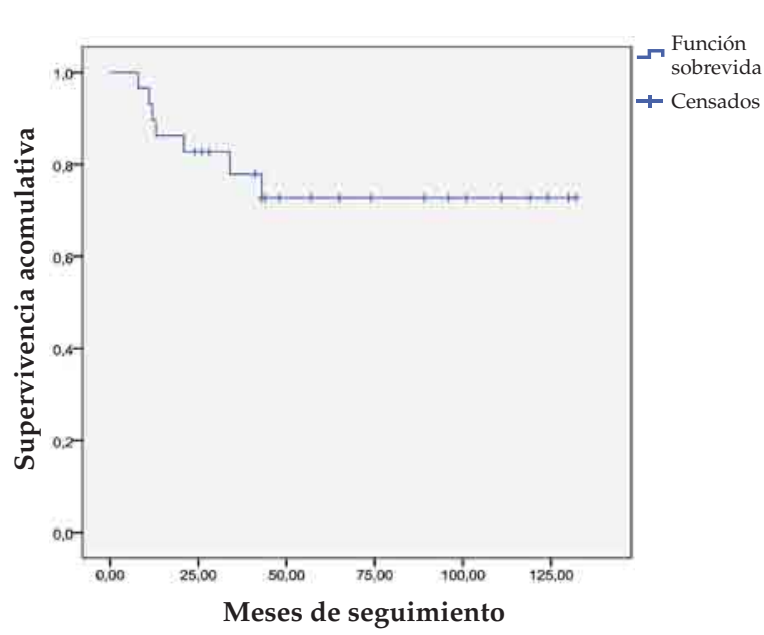

FiguRA 2: A. Imágenes de resonancia magnética nuclear que evidencian una masa heterogénea en hueco popliteo.

B. Pieza quirúrgica resecada. C. Preparado histológico diagnosticado como sarcoma sinovial bifásico.
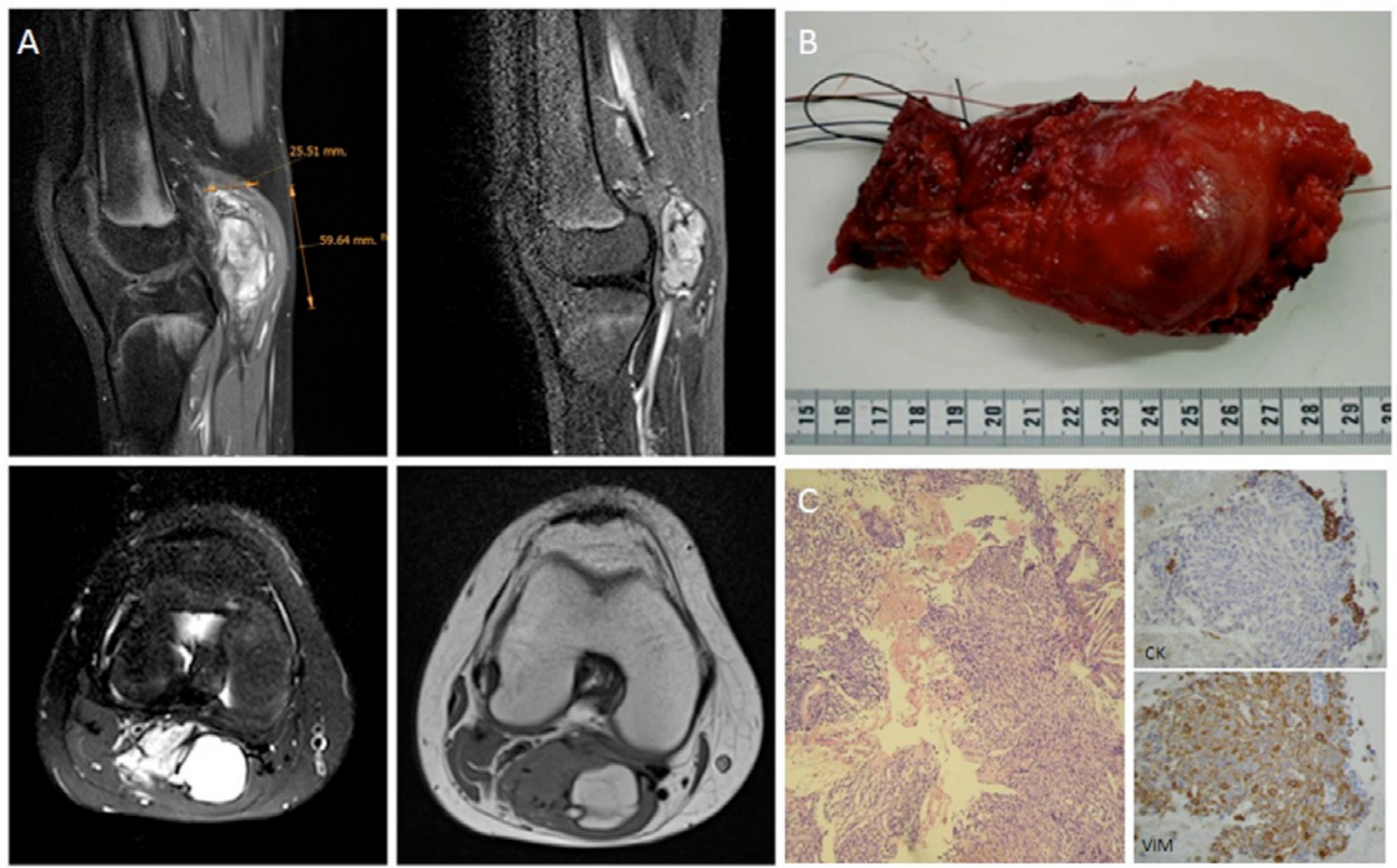
La terapia adyuvante, tanto radioterapia como quimioterapia, no resultó ser un factor pronóstico positivo en términos de supervivencia global o recurrencia $(p>0,05)$.

\section{COMENTARIO}

Los sarcomas pediátricos de partes blandas son infrecuentes y difieren en lo que respecta a su tratamiento y pronóstico en comparación con los tumores de partes blandas de los adultos. En particular, el subtipo NRMS presenta características propias, que lo hacen diferente del subtipo RMS, principalmente por su heterogeneidad, amplia variedad histológica y comportamiento clínico. ${ }^{5,6}$ Los trabajos publicados en la literatura sobre sarcomas de partes blandas en población pediátrica son escasos y, en general, analizan en forma conjunta estos dos subtipos. ${ }^{2,8}$

En nuestra serie, presentamos 29 pacientes, todos con diagnósticos de tumores de partes blandas del tipo NRMS, tratados quirúrgicamente por un mismo equipo quirúrgico. Se describió una supervivencia del $72 \%$ a los 5 años, similar a los resultados antes reportados..$^{9,10}$

La resección quirúrgica ha sido descrita como el pilar fundamental en el tratamiento de este grupo de tumores, orientada a obtener márgenes libres del tumor. ${ }^{2,10-12}$ En 1999, Blakely y col., reportaron la importancia de lograr un margen libre mayor de $1 \mathrm{~cm}$, ya que reduce significativamente la incidencia de recurrencia local. ${ }^{5}$ Ferrari y col. remarcan la importación de garantizar márgenes libres de enfermedad en el tratamiento de este grupo de sarcomas. ${ }^{11} \mathrm{En}$ nuestra serie, siempre se ha tratado a este grupo de tumores con resecciones oncológicas, si bien, en algunos casos, debido al tamaño tumoral y a la zona anatómica localizados, no pudieron asegurarse márgenes amplios.

La radioterapia, como tratamiento adyuvante, ha resultado ser una estrategia de tratamiento satisfactoria para reducir el riesgo de recurrencia local. ${ }^{5,6,11}$ Una de la últimas series publicadas sobre sarcomas de partes blandas en pediatría pertenece a Sawamura y col., quienes han presentado 98 pacientes menores de 18, tratados con cirugía. Se reportó un menor índice de recurrencia local en aquellos pacientes que fueron tratados con radioterapia. Sin embargo, han incluido tanto tumores de tipo RMS como NRMS, sin hacer ninguna diferencia entre los distintos grupos. ${ }^{10}$ Spunt y col., analizaron los factores pronósticos relacionados con los tumores del tipo no rabdomiosarcomas. Han reportado como factores pronósticos positivos para evitar el desarrollo de recurrencia local la localización periférica, la utilización de radioterapia como terapia adyuvante y márgenes oncológicos posquirúrgicos oncológicos libres.

Los posibles efectos secundarios de la radioterapia son un aspecto para tomar en consideración. Son muchos los autores que prefieren restringir su uso en este grupo etario si se han obtenido márgenes libres al momento de la resección. En nuestra serie, no hemos reportado complicaciones secundarias a la radioterapia al momento del último control. Posiblemente, este factor se relacione con los avances tecnológicos asociados a la radioterapia de intensidad modulada. Con respecto a la quimioterapia, si bien representan un factor importante para los tumores del tipo RMS, los tumores NRMS se caracterizan por presentar mayor resistencia. ${ }^{2,11}$ Veinticinco pacientes en nuestra serie recibieron quimioterapia como tratamiento adyuvante; sin embargo, no hemos encontrado diferencias en términos de supervivencia o recurrencia local entre los pacientes tratados con radioterapia, quimioterapia o con cirugía únicamente. El tamaño tumoral $(>5 \mathrm{~cm})$, así como el margen quirúrgico positivo, han sido dos factores que fueron reportados como negativos sobre los resultados oncológicos de los pacientes. 5,6 Otro de los puntos analizados en la literatura es la localización tumoral. Los tumores centrales presentan una peor supervivencia y un mayor índice de recurrencia local en comparación con los tumores de las extremidades. ${ }^{6}$ Es importante remarcar que la posibilidad de realizar una amputación como tratamiento de rescate en los tumores localizados en las extremidades, así como la mayor complejidad técnica para la resección a los sarcomas localizados en pelvis, raquis o tronco, pueden ser las causas que expliquen su peor pronóstico. Particularmente en nuestro estudio, no hemos podido encontrar diferencias significativas en términos de supervivencia entre estos dos grupos.

La presencia de metástasis al momento del diagnóstico o su desarrollo después del tratamiento quirúrgico han afectado de manera significativa la supervivencia de nuestros pacientes. Coincidentemente con la literatura, ha sido el factor de peor pronóstico en nuestra serie. ${ }^{13}$ Hemos observado que la recurrencia local ha sido un factor que aumentó la incidencia de metástasis; es por eso por lo que consideramos de relevancia el tratamiento quirúrgico orientado a lograr 
márgenes amplios en las resecciones. El grado histológico ha resultado otro factor pronóstico determinante en nuestra serie.

El bajo número de nuestra serie, por lo infrecuente de este tipo de sarcomas, puede ser un factor determinante para no poder obtener resultados estadísticos concluyentes en muchos de los factores analizados. Por lo tanto, es relevante realizar a futuro trabajos multicéntricos que permitan aumentar el número de casos.

Conocemos las limitaciones de esta serie por tratarse de un trabajo retrospectivo, con un bajo número de pacientes, heterogeneidad de variedades histológicas y un corto tiempo de seguimiento. Sin embargo, evalúa una patología muy infrecuente, poco reportada en la literatura y con un análisis de los resultados oncológicos del subgrupo NRMS en particular.

\section{CONCLUSIÓN}

Los sarcomas de partes blandas del subtipo NRMS en pacientes pediátricos representan una entidad infrecuente. La recurrencia local y las metástasis son factores de pronóstico negativo para la supervivencia de estos pacientes.

\section{REFERENCIAS}

1. Linabery AM, Ross JA. Childhood and adolescent cancer survival in the US by race and ethnicity for the diagnostic period.1975-1999. Cancer 2008;113(9):2575-96.

2. Loeb DM, Thornton K, Shokek O. Pediatric soft tissue sarcomas. Surg Clin North Am 2008;88(3):615-27, vii.

3. Ferrari A, Dileo P, Casanova M, Bertulli R, et al. Rhabdomyosarcoma in adults. A retrospective analysis of 171 patients treated at a single institution. Cancer 2003;98(3):571-80.
4. Sultan I, Qaddoumi I, Yaser S, Rodríguez-Galindo C, et al. Comparing adult and pediatric rhabdomyosarcoma in the surveillance, epidemiology and end results program, 1973 to 2005: an analysis of 2,600 patients. J Clin Oncol 2009;27(20):3391-7.

5. Blakely ML, Spurbeck WW, Pappo AS, Pratt CB, et al. The impact of margin of resection on outcome in pediatric non rhabdomyosarcoma soft tissue sarcoma. J Pediatr Surg 1999;34(5):672-5.

6. SpuntSL, Poquette CA, Hurt YS, Cain AM, et al. Prognostic factors for children and adolescents with surgically resected non rhabdomyosarcoma soft tissue sarcoma: an analysis of 121 patients treated at St Jude Children's Research Hospital. J Clin Oncol 1999;17(12):3697-705.

7. Guadagnolo BA, Zagars GK, Ballo MT, Strom SS, et al. Mortality after cure of soft-tissue sarcoma treated with conservation surgery and radiotherapy. Cancer 2008;113(2):411-8.

8. Marcus KC, Grier HE, Shamberger RC, Gebhardt MC, et al. Childhood soft tissue sarcoma: a 20 -year experience. J Pediatr 1997;131(4):603-7.

9. Spunt SL, Hill DA, Motosue AM, Billups CA, et al. Clinical features and outcome of initially unresected non metastatic pediatric non rhabdomyosarcoma soft tissue sarcoma. J Clin Oncol 2002;20(15):3225-35.

10. Sawamura C, Springfield DS, Marcus KJ, Perez-Atayde $\mathrm{AR}$, et al. Factors predicting local recurrence, metastasis, and survival in pediatric soft tissue sarcoma in extremities. Clin Orthop Relat Res 2010;468(11):3019-27.

11. Ferrari A, Miceli R, Rey A, Oberlin O, et al. Non-metastatic unresected paediatric non-rhabdomyosarcoma soft tissue sarcomas: results of a pooled analysis from United States and European groups. Eur J Cancer 2011;47(5):724-31.

12. Pratt CB, Maurer HM, Gieser $P$, Salzberg A, et al. Treatment of unresectable or metastatic pediatric soft tissue sarcomas with surgery, irradiation, and chemotherapy: a Pediatric Oncology Group study. Med Pediatr Oncol 1998;30(4):201-9.

13. Pappo AS, Rao BN, Jenkins JJ, Merchant T, et al. Metastatic non rhabdomyosarcomatous soft-tissue sarcomas in children and adolescents: the St. Jude Children's Research Hospital experience. Med Pediatr Oncol 1999;33(2):76-82. 